\title{
INJECTING RHIC FROM THE BROOKHAVEN TANDEM VAN DE GRAAFF*
}

\author{
J. Benjamin, C. Carlson, I. Feigenbaum, M. Manni, D.B. Steski ${ }^{\#}$, P. Thieberger \\ AGS Department, BNL, Upton, NY, 11973, USA
}

\begin{abstract}
In preparation for the start of experiments using the Relativistic Heavy Ion Collider (RHIC) at Brookhaven National Laboratory (BNL), several significant upgrades and improvements were made at the Tandem Van de Graaff. Ion source developments have increased the beam current injected into the tandem, while improvements in foil manufacture and quality control have resulted in better transmission and longer lifetimes for the carbon stripper foils. An oscillating stripper foil chamber has been successfully operated in the high voltage terminal. These improvements have increased the amount of gold ions available from the tandem to $9 \times 10^{9}$ ions per pulse, which is a factor of three increase in the last 3 years.
\end{abstract}

A second tandem Van de Graaff is presently being upgraded and connected to the tandem-to-booster transfer line. Once the upgrade is complete both tandems will have the ability to deliver ions to RHIC. It will also be possible for one tandem to inject RHIC while the other provides beam to a local target room, or to an application facility at the AGS Booster.

\section{INTRODUCTION}

The two MP Tandem Van de Graaffs (MP6 and MP7) at Brookhaven National Laboratory (BNL) began providing heavy ions to local target rooms in 1970. In 1986 a transfer line approximately 680 meters long allowed the tandems to directly inject heavy ions into the Alternating Gradient Synchrotron (AGS) for further acceleration. With the completion of the Booster Synchrotron in 1992 the transfer line was extended and gold ions could be accelerated in both the Booster and the AGS. In May of 1999, the Tandem Van de Graaffs will enter a new era when MP7 begins delivering gold ions for the Relativistic Heavy Ion Collider (RHIC).

The accelerating scheme for RHIC is shown in figure 1. Negative ions are produced by a cesium sputter ion source and then are accelerated to the terminal of the tandem. There the beam passes through a $2 \mu \mathrm{g} / \mathrm{cm}^{2}$ carbon stripper foil and is stripped to the +12 charge state. The gold ions are accelerated back to ground potential where they are stripped to the +31 or +32 charge state using an

" This work performed under the auspices of the U.S. Department of Energy

"Email: Steski@bnl.gov object stripper foil approximately $13 \mu \mathrm{g} / \mathrm{cm}^{2}$ thick. This beam is then transported to the Booster where it is accelerated further, stripped, transferred to the AGS where it is again accelerated and stripped, and finally fills the two counter-rotating rings of RHIC.

\section{RHIC ACCELERATION CONFIGURATION}

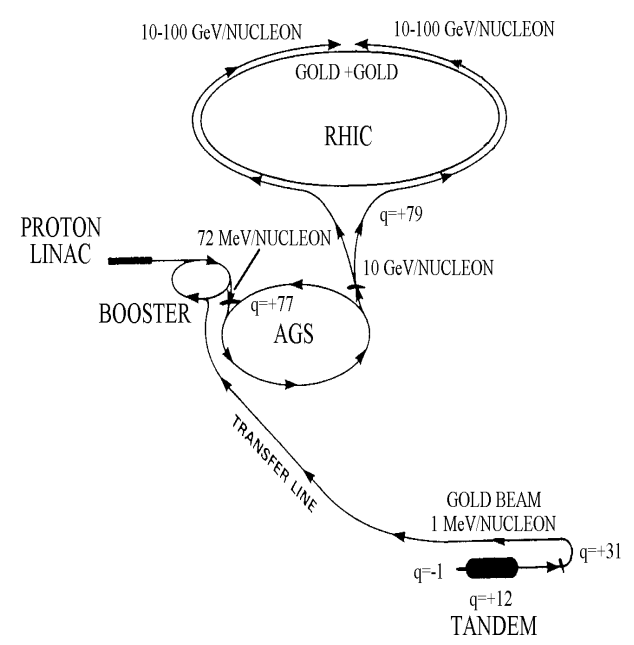

Figure 1: RHIC Acceleration Configuration

The Booster requires short pulses of high charge state beams at approximately $1 \mathrm{MeV} /$ nucleon. To achieve this the terminal of MP7 is run at $14 \mathrm{MV}$ and the cesium sputter ion source is operated in a pulsed beam mode[1]. Pulsing the ion source allows several orders of magnitude higher instantaneous beam currents to be injected into the tandem than conventional DC operation without damage to the accelerator. The pulse length from the ion source can be as long as $2 \mathrm{msec}$ however it is usually operated at between $500 \mu \mathrm{sec}$ and $1000 \mu \mathrm{sec}$. For the AGS fixed target program the acceleration cycle typically calls for the tandem to deliver a pulse once every $3.5 \mathrm{sec}$. In the collider mode of operation the requirements are to deliver 4 pulses separated by $200 \mathrm{msec}$ once every $3.5 \mathrm{sec}$ for approximately 1 minute, until both rings of the collider are full[2]. The next request to fill the rings should occur on the order of 10 hours later. 
The experience gained by injecting heavy ions into the AGS and the Booster has led to a number of improvements in the last few years. The improvements that have been made or are planned in the near future are discussed below.

\section{ION SOURCE IMPROVEMENTS}

There has been a research and development effort for a number of years to improve the performance of the cesium sputter sources. The results of this effort can be clearly seen in figure 2, which shows the increase in peak beam intensity versus year. There has been a three-fold increase in the peak intensity available to the booster in the past three years. The increase has been mainly a result of efforts to reduce the large space charge forces experienced by the ion beam.

One of the methods used to reduce the space charge forces was to increase the energy of the ion beam. Beginning in 1996 the original General Ionix 860 [3] source was replaced by the Peabody Scientific PSX-120 [4] ion source. This allowed the ion source beam energy to be increased from $25 \mathrm{keV}$ to $32 \mathrm{keV}$. The highest demonstrated current was $90.4 \mu \mathrm{A}$ of $\mathrm{Au}^{+31}$ when injecting at a beam energy of $35 \mathrm{keV}$. For a pulse length of 500 $\mu$ sec this corresponds to $9 \times 10^{9}$ ions per pulse, which is double the intensity specified in the RHIC Design Manual[5].

Although limited research has been done at higher injection energies, it appears possible to deliver approximately $120 \mu \mathrm{A}$ of $\mathrm{Au}^{+31}$ or $12 \times 10^{9}$ ions per pulse when using an extraction voltage of $50 \mathrm{kV}$. To operate reliably at $50 \mathrm{kV}$ some minor modifications to the ion source will have to be made. The effects of these modifications on the beam optics of the ion source will have to be investigated.

Another improvement made was the insertion of an einzel immediately downstream of the ion source. First tried in 1997, this compensated for the blow up of the low energy ion beam and allowed higher beam currents to be transported to the entrance of the tandem.

There has also been a growing interest in accelerating uranium beams in RHIC. Unfortunately uranium does not form an abundant negative ion. Therefore the effort has concentrated in finding a suitable molecule that readily forms a negative ion. Preliminary results indicate that UO- is formed in sufficient quantity to provide usable beams. Using a uranium sputter target with a hole drilled in the middle to allow oxygen to be bled onto the surface and a unique pulsing scheme, a pulse with a peak intensity of $34 \mu \mathrm{A}$ of UO- has been generated on the ion source test bench. Scaling this number to MP7 and using the efficiencies generated from the gold beam, this should generate a pulse with a peak intensity of $25 \mu \mathrm{A}$ of $\mathrm{U}^{+31}$. For a $600 \mu \mathrm{sec}$ pulse, that would be $3 \times 10^{9}$ ions per pulse. Although this intensity is much less than the gold beam, it should be usable for RHIC. Because the research to produce a uranium beam for RHIC has just begun, it is hoped that further increases will be possible by reducing the space charge forces of the beam and by finding a more abundant negative molecule.

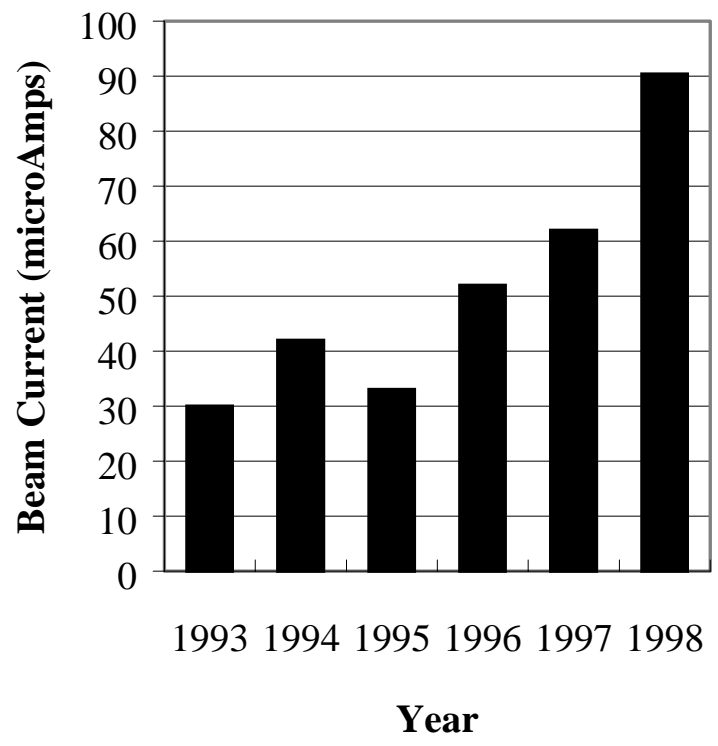

Figure 2: Peak Gold Intensity vs Year

\section{IMPROVEMENTS IN STRIPPER FOILS}

The thickness and lifetime of the carbon stripper foils have a profound effect on the quality and quantity of the ion beam delivered to the booster.

The thickness of the foils at the center terminal determines the transmission of the beam through the tandem. The thickness needs to be as close to $2 \mu \mathrm{g} / \mathrm{cm}^{2}$ as possible. To insure that the foils are of the proper thickness they are tested using an optical densitometer prior to being installed in the accelerator. A program is also under way to test the foils offline by looking at the energy lose through the carbon foils of a low energy $\mathrm{He}+$ beam.

Once RHIC is operating with a beam survival of 10 hours, the lifetime of the terminal stripper foils will become less of a problem, as each foil will last for several days. However, during the setup phase, when high intensity pulses will be required every 3 seconds, the stripper foil lifetime will be very important. To improve the lifetime, an oscillating foil holder containing 300 foils has been installed in the terminal of MP7. The dimensions of the terminal foils were increased to $2.2 \mathrm{~cm}$ by $1 \mathrm{~cm}$ to allow for the $1.25 \mathrm{~cm}$ movement of the oscillator. The period of the oscillator was approximately 1 minute when it was first used during the 1998 heavy ion run. The average oscillating foil lifetime was approximately 6 
hours, compared to the 2-hour lifetime of the stationary foils.

Although the object foils have a longer lifetime, an oscillating foil holder has also been installed at this position. The object foil oscillator is not operated continuously but rather is occasionally moved to a different spot. This has allowed object foils to last in excess of 24 hours.

\section{MP6 UPGRADE AND THE INTERCONNECTING LINE}

Presently the MP6 tandem Van de Graaff is undergoing the same upgrade as was completed on MP7[6] in the early 1980's. One of the major upgrades is to replace the existing accelerator tubes with longer tubes. Seven of the present $190 \mathrm{~cm}$ accelerator tubes have been replaced with $223 \mathrm{~cm}$ tubes, while the first tube has been replaced with a $244 \mathrm{~cm}$ tube of which the first $40 \mathrm{~cm}$ is half gradient. This has increased the active length of the tandem by $22 \%$ and will increase the maximum operating voltage.

Some of the other improvements to MP6 include more vacuum pumping along the accelerator tubes, the addition of 3 more pelletron chains for charging, and improved voltage dividers for the accelerator tubes and the support column. These improvements will make the two machines almost identical and allow MP6 to reach a terminal voltage of $14 \mathrm{MV}$ reliably.

Once the upgrade is completed an inter-connecting line will bring the ion beam from MP6 to the existing MP7 beamlines. The interconnecting line consists of four 25-degree dipoles and one 90-degree dipole. The first two 25-degree dipoles will deflect the ion beam around MP7. If the beam is required in one of the local target rooms the 90-degree magnet will bend the beam into the existing image leg of MP7. However, if the beam is being used to inject RHIC then the other two 25-degree dipoles will deflect the beam into the MP7 object leg and into the tandem-to-booster transfer line.

The inter-connecting line will allow MP6 beam to be delivered to local target rooms at the same time that MP7 beam is being injected into RHIC. It will also allow the beams from MP6 to be diverted to RHIC if needed.

The fourth 25-degree dipole that is shared by the inter-connecting line and the MP7 object leg is a fast switching magnet. This will allow one beam to be delivered to RHIC from MP7 and then 3 seconds later a different species to be delivered to RHIC from MP6. This has uses for asymmetric ion species in RHIC with the same rigidity and will also allow different beams to be delivered to the Booster Application Facility.

\section{CONCLUSIONS}

The Brookhaven tandem Van de Graaffs have already surpassed the RHIC design specification and there are plans to increase the intensity further. The upgrade of
MP6 and the interconnecting line are under construction and when completed will allow the target room user program to operate at the same time as RHIC. The tandems have proved themselves to be versatile reliable injectors for the AGS synchrotrons and with the number of recent improvements and upgrades they should prove to be just as reliable for RHIC.

\section{REFERENCES}

[1] Thieberger P., McKeown M., and Wegner H.E., IEEE transactions on Nuclear Science, NS-30 No. 4, 2746-2748, (1983)

[2] RHIC Design Manual, http://www.agsrhichome.bnl.gov/NTshare/rhicdm/

[3] Distributed by High Voltage Engineering Europa, P.O. Box 99 AB Amerfort, The Netherlands

[4] Distributed by Peabody Scientific, Peabody, Ma, 01960, USA

[5] RHIC Design Manual, http://www.agsrhichome.bnl.gov/NT$\underline{\text { share/rhicdm/ }}$

[6] Thieberger P., Nucl. Instr. And Meth. 220 (1984) 45-53 